management, and 3) saving lives at the accident site. Each subgoal included 20 to 29 additional subgoals at up to eight different levels, with associated specific tasks and plans.

Conclusion: The HTA provided a unified structure for the complex task of responding to a major train accident in cold climate and inaccessible terrain. One specific benefit of the HTA was that it provided an overview of organizational interdependencies, and can serve as a tool when developing and streamlining response plans to major incidents.

Prehosp Disaster Med 2017;32(Suppl. 1):s19-s20

doi:10.1017/S1049023X17000723

\section{Hospital Disaster Victim Registration: A National Standard} in Belgium

Christel Hendrickx ${ }^{1}$, Wim Hermans ${ }^{2}$, Marcel Van Der Auwera ${ }^{2}$, Marc Sabbe ${ }^{1}$

1. Emergency Department, University Hospitals Leuven, Leuven/ Belgium

2. Federal Ministry of Public Health, Brussels/Belgium

Study/Objective: Development of a National standard for victim registration.

Background: During disasters, hospitals are overwhelmed with questions about potential victims, one of the most disturbing elements during the initial response phase. However, there is a need for early and accurate victim information for the relatives about identification, hospital location, and severity. The Belgian authorities took the initiative to develop and test such a system in a pilot hospital.

Methods: The study used a mixed-method design. Initially, a structured questionnaire was developed and sent to all EDs of the Flemish part of Belgium. Out of the questionnaire responses, an exchange disaster victim identification system was distillated. The feasibility of this system was tested in a pilot hospital using a command post exercise. The qualitative part consisted of semi-structured interviews to analyse the structure and actions of the hospital staff within the system.

Results: The response rate to the questionnaire was $75 \%$. The awareness of the need of such a system was extremely high - high $(\mathrm{x}-\mathrm{y})$ : ED head nurses $(68 \%-30 \%)$, disaster coordinators $(62 \%-22 \%)$, ED nurses $(44 \%-42 \%)$, emergency physicians $(24 \%-38 \%)$, and other ED personnel $(16 \%$ - 38\%). A command post exercise demonstrated the feasibility of the developed standardized exchange disaster victim identification system. These structures need sufficient and additional personnel; the observations and interviews provided evidence that there is still room for process improvement.

Conclusion: There is a need for a standardized national disaster victim identification system, adapted to the context of hospitals. A national partnership was developed concerning such a system with standard guidelines and usable registration tools. The collaboration agreement will be effectively implemented in all Belgian hospitals. Testing this national system at a pilot hospital was an important step in creating this generic document for Belgium.

Prehosp Disaster Med 2017;32(Suppl. 1):s20

doi:10.1017/S1049023X17000735
Disaster Response Coordination among Disaster Management Organizations in Modern Cities: The Case of Nairobi County, Kenya

Ali A. Wangara

Accident And Emergency, Kenyatta National Hospital, Nairobi/Kenya

Study/Objective: To assess disaster response coordination among disaster management organizations in Nairobi County in the last five years, identify factors affecting, and establish ways of improving disaster response coordination in Nairobi County.

Background: Disaster response coordination ensures access to core information and efficiency of response actions among responding organizations. This helps mitigate against morbidity and mortality that result following disaster events. However, coordination has remained a significant problem during and after each disaster. In Kenya, frequency of disasters has heightened with observable response coordination challenges among the several disaster management agencies specialized in various fields.

Methods: We carried out a cross-sectional study that utilized quantitative and qualitative methods among disaster management organizations in Nairobi County, Kenya. All of the 71 organizations providing ambulance transport, fire-fighting, security, health services, rescue and media were recruited. A respondent drawn from an organization provided information on a self-administered semi-structured questionnaire. Quantitative data was analyzed on SPSS Version 20.0, while qualitative was analyzed thematically. Deductions were drawn from frequencies and proportions of the findings and presented as narrative, tables and figures.

Results: Organizations included media houses, air ambulances, military and humanitarian organizations such as the Kenya Red Cross and St. John's Ambulance, that had a broad approach to response. Majority, 46 (75.4\%), reported interrelating with experience of in-optimal response $(53.2 \%$ responded to $<10$ out of 27 listed emergencies). Factors that affected coordination included age of organizations $\times 2(1)=5.031, \quad P=0.025$, inter-communication $\times 2(1)=$ $34.252, \mathrm{P}<0.001)$, presence of emergency response policy $\times 2(1)=15.149, \mathrm{P}<0.001)$ and knowledge sharing $\times 2(3)=$ 12.921, $\mathrm{P}=0.005)$. To improve coordination, they indicated the need to improve success factors such as positive public response, inter-cooperation, enhanced government role, participation of Non-Governmental Organizations and a fair command system. Others were to prioritize disaster operations by continuous integration and synchronization of disaster plans, symposia and funding.

Conclusion: Organizations had limited inter-relationships with nonoptimal response to emergencies. A major factor was lack of guidelines. There is need for the organizations and county governments to initiate an overall forum, to hold symposia for stakeholders and to draw coordination standards and guidelines. Further research is needed to determine if regular inter-organizational cooperation would improve disaster response coordination in Nairobi County.

Prehosp Disaster Med 2017;32(Suppl. 1):s20

doi:10.1017/S1049023X17000747 